\title{
Dietary supplement use and documentation in a breast cancer survivorship clinic
}

\author{
Julia Silver $^{1} \cdot$ Alyson Goldenberg $^{1} \cdot$ Anne Moore $^{1}$ (i)
}

Received: 26 September 2021 / Accepted: 14 November 2021 / Published online: 24 November 2021

(c) The Author(s), under exclusive licence to Springer Science+Business Media, LLC, part of Springer Nature 2021

\begin{abstract}
Purpose Breast cancer survivors take vitamins and supplements to bolster their general health and to decrease the risk of cancer recurrence. Healthcare providers are frequently unaware of their patients non-prescription supplement use. The aim of this study was to study the type and the documentation of patients' dietary supplements and vitamins in the electronic medical record (EMR).

Methods 50/51 female breast cancer survivors seen over a 7 week period consented to the study. Mean age was 70 and mean years since diagnosis was 13.9. Informed consent and documentation of supplement and vitamin use was obtained by the nurse practitioner the day before the visit. Study data were collected and managed using REDCap electronic data capture tools hosted at Weill Cornell Medicine.

Results Of the 50 study patients, $90 \%$ were taking one or more vitamins and/or supplements (mean $=2.4$, range $=1-9$ ). The most common were Vitamin D, calcium, and vitamin C. Reasons for vitamin and supplement use included the recommendation by their physician or friend and prevention of bone loss or catching a cold. Five patients mentioned immunity or prevention of COVID-19. The patient reported list was compared with the medication list used by multiple providers in the electronic medical record (EMR). None of the 50 study patients had an accurate list of their vitamins and supplements in the EMR.

Conclusion $90 \%$ of the breast cancer survivors in our study were taking dietary supplements for a variety of reasons. None had an accurate list in the EMR. We strongly recommend more attention to accurate and easily accessed vitamin and supplement recording by providers.
\end{abstract}

Keywords Survivorship · Dietary supplements $\cdot$ Breast cancer $\cdot$ Electronic medical record (EMR)

\section{Introduction}

Women and men who have completed "active" treatment for breast cancer continue to worry about the risk of recurrence and the long-term impact of their therapy [1]. Many patients seek information about dietary supplements to improve their general health and to bolster their "immune health" [2]. As defined by the Federal Drug Administration, dietary supplements are "products taken by mouth that contain a 'dietary ingredient.' Dietary supplements include vitamins, minerals, amino acids, herbs and botanicals" [3]. Dietary supplements

Anne Moore

almoore@med.cornell.edu

1 Iris Cantor Breast Cancer Survivorship Program, NewYork Presbyterian Hospital-Weill Cornell Medicine, 425 East 61st Street, 10th floor, New York, NY 10065, USA are the primary complementary and alternative therapy used by cancer survivors in the USA [4]. Studies addressing whether these dietary supplements are beneficial or harmful show inconsistent findings, and many are inconclusive [5]. Physicians are frequently unaware of their patients' use of dietary supplements. The electronic medical record (EMR) poorly reflects non-prescription dietary supplement use [6].

We studied dietary supplements taken by fifty sequential patients in the Iris Cantor Breast Cancer Survivorship Program at Weill Cornell Medicine NewYork-Presbyterian Hospital. The three aims of this study were to determine which dietary supplements patients were taking and for what reason, to compare the patient's list with the existing documentation in the EMR and to create an accurate list of the dietary supplements in the EMR for future reference. 


\section{Patients and methods}

All patients of the Breast Cancer Survivorship Program seen between May 5th and June 24th, 2020 were invited to participate in the study prior to their routine annual visit. The eligibility included the absence of metastatic disease and willingness to provide informed consent. Informed consent was obtained from all individual participants included in the study. The nurse practitioner (NP) conducted a pre-visit telephone call to invite patients to participate in the study. The NP asked the study participants about the names, doses, and reasons for taking the supplements. The data obtained by the NP was put into each patient's EMR and reviewed during the subsequent clinic visit. Study data were collected and managed using REDCap electronic data capture tools hosted at Weill Cornell Medicine [7]. We recorded the supplements that patients were taking under the medication section of the EMR using free text for the majority of the supplements. We also included the reason for taking each supplement in the patient's own words. The study was approved by the Weill Cornell Medicine IRB. Informed consent was obtained from all individual participants included in the study. Due to Covid-19, telemedicine was used by the nurse practitioner for 48 of 50 visits to explain the study and obtain informed consent. The physician visit was also conducted through telemedicine for 48 of 50 patients.

\section{Results}

Fifty-one women were scheduled for their routine annual survivorship visit during this study period. Fifty of the 51 women, ages 46 to 87 (mean age 70) agreed to participate in the study. The average number of years since the diagnosis of breast cancer was 13.5 (range 0-34). Of the 50 study participants, the mean number of dietary supplements taken was 2.4 (range 0-9). Five patients were taking no supplements, 36 patients were taking 1-3 supplements, 7 patients were taking 4-6 supplements, and 2 patients were taking
7-9 supplements. The four most common supplements taken were vitamin D (41 patients), calcium (21 patients), vitamin C (12 patients), and a multivitamin (8 patients). Twenty-four additional dietary supplements were taken by less than $10 \%$ of the patients (Table 1).

Patients reported a variety of reasons for their dietary supplement use. Some patients stated the supplement was recommended by their medical provider or a friend, some did not know why they were taking a specific supplement, and some reported that they had "always taken it". Many stated that the supplement was a preventative measure against bone loss or catching a cold. Five patients mentioned immunity or prevention of COVID-19 (Table 2).

The majority of the participants had multiple providers who entered medications in the EMR prior to the study visit. We compared the patient's accurate, updated dietary supplement list recorded by the study NP with the medication list in the EMR documented by previous providers. Of the fifty participants in our study, none had an accurate list of dietary supplements in their EMR prior to the study visit day.

Table 2 Patient reasons for taking dietary supplements

Bone and joint health: 23

Physician recommendation: 10

General health: 6

"Always taken vitamins": 5

Hair and/or nail strength: 5

Strengthen immune system: 5

Heard or read it's good for you: 5

Not sure: 3

Miscellaneous (insomnia, bladder symptoms, advancing age): 2 each

One each:

Lung health

Macular degeneration

Digestion

Constipation

Anti-inflammatory purposes

"Because it is good for my heart"
Table 1 Dietary supplements taken by less than $10 \%$ of patients

\begin{tabular}{|c|c|c|c|c|}
\hline Vitamins & Minerals & Herbs and botanicals & Hormones & Miscellaneous \\
\hline Biotin & Magnesium & Black elderberry & Melatonin & Coenzyme Q10 \\
\hline Folic Acid & Selenium & Chinese Herbal Tea & & Fish oil \\
\hline Lutein & Zinc & Coxamin & & Glucosamine \\
\hline Vitamin B12 & & Cranberry & & Probiotics \\
\hline Vitamin E & & Curcumin & & Sorbitol \\
\hline \multirow[t]{4}{*}{ Vitamin K } & & Ginseng Root Powder & & \\
\hline & & Rosemary & & \\
\hline & & Shiitake Extract & & \\
\hline & & Turmeric & & \\
\hline
\end{tabular}




\section{Discussion}

Dietary supplement use among cancer survivors is common. Du et al. report that $70.4 \%$ of cancer survivors take dietary supplements, compared to $50 \%$ of the general population [8]. Breast cancer survivors consistently report the highest use of dietary supplements compared to survivors of other cancers $[2,6]$. Ninety percent of our study participants were taking one or more supplements (mean 2.4, range 1-9).

Breast cancer survivors take dietary supplements for various reasons. Studies show that supplement use is often tied to the hope of preventing recurrence as reported by the LEAN study $[9,10]$. In our study, prevention of cancer recurrence was not cited as a reason for dietary supplement use. The most common reason for taking supplements among our patients was bone health. We think this difference between our patients and those of the LEAN study reflects the maturity of our group with a median age of 70 and a mean time since diagnosis of almost 14 years. This compares to the LEAN study, where the median age was 58 years and the time since diagnosis was 2.9 years [11]. The younger patients with a more recent diagnosis of breast cancer may be more concerned about trying to prevent recurrence with supplement use than our older population with an average of 13.5 years since diagnosis. Other reasons for supplement use among our study participants included "I read it was good for me" or "to help my hair and nails". Additional reasons included "immune health" and protection against COVID-19. Only one participant mentioned prevention of heart disease as a reason for taking dietary supplements (Table 2). This was surprising as heart disease is the main cause of death in women (12).

In our study, none of the participants had an accurate list of dietary supplements in their EMR. Studies by others confirm that the majority of physicians are not aware of their patients' use of dietary supplements [5]. This may be due to physicians and other medical providers not asking patients about supplement use, or physician belief that this information is not important enough to be recorded in the medical record. In addition, physicians may not feel competent to counsel patients on supplement use. We also believe patients are hesitant to tell their medical providers about supplement use unless specifically asked. Finally, electronic medical records do not provide a simple way for the busy medical provider to record the dietary supplements at each visit.

A review of an accurate list of supplements at the time of the survivorship visit allows the medical oncologist, the oncology nurse practitioner, or the primary care provider to consider possible deleterious interactions between supplements and medications such as tamoxifen and aromatase inhibitors [11]. Furthermore, discussion of supplements will help patients feel confident that the investment they are making in their health is worthwhile. This conversation may also lead to a discussion of topics of general health such as osteoporosis and, importantly, fear of cancer recurrence. A simple patient guide that outlines the pros and cons of supplement usage, such as the FDA 101 guide may be provided for patients to widen their knowledge base [3]. In addition, an accurate list of dietary supplements in the EMR may be important for health care research in the future. This would not be possible at the current level of vitamin and supplement documentation in the EMR.

Our study of supplement use in this cohort of breast cancer survivors confirms Velicer et al.'s observation that breast cancer survivors commonly take dietary supplements and that their medical providers are unaware of which supplements they are taking [6]. The EMR potentially provides us with innovative ways to gather data entered by the patient and the medical provider about supplement use. Continuing education of medical providers about documentation of dietary supplement use will increase patient satisfaction and ultimately improve patient care.

In conclusion, ninety percent of the fifty breast cancer survivors enrolled in this study reported that they were taking dietary supplements. A total of 28 supplements were reported, four of which were commonly used. No study participant had an accurate list of their dietary supplements in the EMR prior to the day of their study visit. We recommend that all dietary supplements should be recorded and acknowledged. We also recommend that there be a documentation method in the EMR to make this convenient and satisfactory for the medical providers.

Acknowledgements This work was supported by the Iris Cantor Breast Cancer Survivorship Program, NewYork Presbyterian Hospital-Weill Cornell Medicine and the Anne Moore Breast Cancer Research Fund, Weill Cornell Medicine This work was presented as a poster at the San Antonio Breast Cancer Symposium, 2020. Abstract PS9-12: Vitamin and supplement use and documentation in a breast cancer survivorship clinic. Anne Moore, Julia Silver, and Alyson Goldenberg. Cancer Res February 152021 (81) (4 Supplement) PS9-12; https://doi.org/10.1158/ 1538-7445.SABCS20-PS9-12

Author contributions JS-Data management and manuscript preparation and approval; AG-Obtained patient consent, conducted patient interviews, manuscript review and approval; AM-Project supervision, IRB approval, and manuscript preparation and approval.

Funding Iris Cantor Breast Cancer Survivorship Program, NewYork Presbyterian Hospital-Weill Cornell Medicine and the Anne Moore Breast Cancer Research Fund, Weill Cornell Medicine. Weill cornell medical college.

Data availability REDCap (Research Electronic Data Capture) is a free data management software system that is fully supported by the Weill-Cornell Medical Center Clinical Translational Science Center 
(CTSC). It is a tool for the creation of customized, secure data management systems that include Web-based data-entry forms, reporting tools, and a full array of security features including user and group based privileges, authentication using institution LDAP system, with a full audit trail of data manipulation and export procedures. REDCap is maintained on CTSC-owned servers that are backed up nightly and support encrypted (SSL-based) connections. Nationally, the software is developed, enhanced, and supported through a multi-institutional consortium led by the Vanderbilt University CTSA. All data generated or analyzed during this study are included in this published article.

Code availability Not applicable.

\section{Declarations}

Conflict of interest The authors JS, AG, AM declare they have no conflict of interest.

Ethical approval: See 3 approvals attached to the submission. The IRB approved switching from written to verbal consent by telephone due to COVID.

Consent to participate See 3 approvals attached to the submission. The IRB approved switching from written to verbal consent by telephone due to COVID.

Consent for publication Not applicable.

\section{References}

1. Offidani E, Peterson JC, Loizzo J, Moore A, Charlson ME (2017) Stress and response to treatment: insights from a pilot study using a 4-week contemplative self-healing meditation intervention for posttraumatic stress in breast cancer. J Evid Based Complementary Altern Med. https://doi.org/10.1177/2156587217708522

2. Song S, Youn J, Lee YJ, Kang M, Hyun T, Song Y et al (2017) Dietary supplement use among cancer survivors and the general population: a nation-wide cross-sectional study. BMC Cancer. https://doi.org/10.1186/s12885-017-3885-1

3. US Food and Drug Administration, FDA 101: Dietary Supplements (online brochure, updated 7/15/15). Accessed at www.fda. gov/ForConsumers/ConsumerUpdates/ucm050803.htm on May 12, 2021.
4. Bernstein BJ, Grasso T (2001) Prevalence of complementary and alternative medicine use in cancer patients. Oncology (Williston Park) 15:1267-1283

5. Tarn DJ, Paterniti DA, Good JS, Coulter ID, Galliher JM, Kravitz RL et al (2013) Physician-patient communication about dietary supplements. Patient Educ Counsel 91(3):287-294. https://doi. org/10.1016/j.pec.2013.01.021

6. Velicer CM, Ulrich CM (2008) Vitamin and mineral supplement use among US adults after cancer diagnosis: a systematic review. J Clin Oncol 26(4):665-673. https://doi.org/10.1200/JCO.2007. 13.5905

7. Harris PA, Taylor R, Thielke R, Payne J, Gonzalez N, Conde JG (2009) Research electronic data capture (REDCap) - A metadata-driven methodology and workflow process for providing translational research informatics support. J Biomed Inform 42(2):377-381

8. Du M, Luo H, Blumberg J, Rogers G, Chen F, Ruan M et al (2020) Dietary supplement use among adult cancer survivors in the united states. J Nutr 150(6):1499-1508. https://doi.org/10.1093/ jn/nxaa040

9. Nguyen T, Harrigan M, Mcgowan C, Hood A, Fangyong Li, Cartmel B et al (2021) Abstract PS8-09: dietary supplement use in a healthy eating and exercise lifestyle intervention in breast cancer survivors: the lifestyle exercise and nutrition (LEAN) study. Cancer Res. https://doi.org/10.1158/1538-7445.SABCS20-PS8-09

10. DePolo J. (Host). (2020, Dec 14). SABCS 2020: Supplement Use After Breast Cancer [Audio podcast episode]. In The Breastcancer.org Podcast. Breastcancer.org. https://www.breastcancer.org/ community/podcasts/sabcs2020-supplements

11. Nguyen T, Harrigan M, McGowan C, Hood A, Li F, Cartmel B, Ferrucci L, et al. 2020 Dietary Supplement Use in a Healthy Eating and Exercise Lifestyle Intervention in Breast Cancer Survivors: The Lifestyle Exercise and Nutrition (LEAN) Study. Poster session presented at: San Antonio Breast Cancer Virtual Symposium Dec 8-11; San Antonio, TX.

12. Benjamin EJ, Muntner P, Alonso A, Bittencourt MS, Callaway CW, Carson AP et al (2019) Heart disease and stroke statistics-2019 update: a report from the American heart association. Circulation. https://doi.org/10.1161/cir.0000000000000659

Publisher's Note Springer Nature remains neutral with regard to jurisdictional claims in published maps and institutional affiliations. 